\title{
LES ALGUES D'EAU DOUCE ET LEUR INTÉRET EN PISCICULTURE
}

\author{
par \\ P. VIVIER \\ et \\ E. MANGUIN \\ (Station Centrale d'Hydrobiologie appliqué.) \\ (Museum national d'Histoire naturelle.)
}

Les innombrables Algues, la plupart microscopiques, qui peuplent nos eaux douces, ont un intérêt puissant en pisciculture; ce sont elles qui forment presque entièrement la nourriture des animaux du Plancton et du Benthos, notamment celle des Rotifères et des Entomostracés, eux-mèmes recherchés des poissons; Diffesbach notamment a publié à ce sujet un graphique (1) des plus suggestifs qui montre la relation étroite existant entre la quantité de Plancton et le nombre de Rotifères dans un mème volume d'eau ; les deux courbes qui expriment le nombre d'individus en fonction du temps pour chacun des deux groupes sont sensiblement parallèles. Sans les Algues d'eau douce, les Poissons ne sauraient donc vivre, à moins de recevoir une nourriture artificielle, viande ou farineux suivant les espèces, c'est dire l'importance pratique de l'Algologie.

Celte science nous apprend, entre autres, quels sont les meilleurs milieux de culture pour tels ou tels groupes d'Algues : on conçoit que dans les immenses vases de l'etri que forment les étangs, il soit possible, théoriquement, d'en diriger en quelque sorte la production par l'addition de produits chimiques semblables à ceux utilisés dans les cultures de laboratoire; les engrais dont le rôle bienfaisant en pisciculture est prouvé, surtout depuis les expériences de Wirten à Willenbach (2) trouvent dans l'Algologie l'explication de leur efficacité ; ce sont eux qui apportent à ces innombrables végétaux les matières minérales dont ils ont besoin ; ce sont eux qui créent le milieu de culture favorable dont bénéficiera en définilive le Poisson.

Aussi n'avons-nous pas cru inutile d'insister un peu longuement sur l'écologic et la biologie des Algues dans les quelques pages qui cont suivre.

(1) L'un de nous a reproduit ce graphique dans : - "Les Principes de la Limnologie ", - Bulletin Français de Pisciculture, no 70, Avril 1934, p. 279.

(2) P. Vrvieg: --- La fumure des étangs. - Journal d'A gricullure pratique, 1934. 


\title{
I. - LES ALGLES D'EAU DOUCE
}

\author{
par E. MANGUIN \\ (Musetum national d'Histoire naturelle.) \\ I. — GÉNÉRALITÉS BTOLOGIQUES
}

A l'encontre de tous les animaux, depuis les Protozoaires jusquaux Vertébrés, qui ne savent pas utiliser l'énergie lumineuse du soleil pour la synthèse de leur propre substance vitale, les plantes vertes, que le parasitisme n'a pas dégradées, se servent de cette énergie pour édifier leur protoplasma, exclusivement à parlir d'éléments chimiques puisés dans le milieu cosmique : eau, gaz carbonique, nitrates, sels ammoniacaux. Leur extraordinaire pouvoir de synthèse est capable d'édifier, à partir de ces éléments chimiques simples, un rrand nombre de molécules organiques, ou groupements chimiques extrêmement complexes.

Que ce soit sur la terre ou au sein des caux, la plante joue le même rôle créateur. Ainsi pour le sujet qui nous intéresse, la majorité des Algues est holophyte (autotrophe) (1), en un mol, capable de se nourrir exclusivement aux dépens du gaz carbonique de l'atmosphère. Grâce à ce pouvoir de photosynthèse, lles Algues préparent une vie supérieure en devenant la proie des animaux microscopiques, lesquels à leur tour servent d'aliments à d'autres plus gros, ainsi de suite jusqu'à l'ultime terme de l'échelle animale.

Le phytoplancton, c'est-à-dire l'ensemble de tous les représentants de la végétation algale vivant au seịn des eaux douces, compose la presque totalité de la nourriture du plancton animal ou zooplancton, principalement celle des Rotifères et des Crustacés. Ceux́ci sont, à leur tour, la base des éléments nutritifs des Poissons qui peuplent les étangs, les lacs et les rivières. Lion peut aisément se rendre comple de la puissance productive du phytoplancton, par les observations de BIRGe et Judar (2) sur la qualité nutritive du plancton du Lac Mendota dans le Wisconsin; ces auteurs estiment qu'un hectare de surface dès eaux de ce lac intérieur donne 240 kilos de matière organique sèche et portent à $2.800 \mathrm{kilos}$ la production annuelle pour une même unité de surface.

La grande classe des Algues, qui comprend tous les thallophytes pourvus de chlorophylle, qu'ils soient marins ou d'eau douce, se divise en trois sous-classes, selon les caractères des pigments élaborés par les chloroplastes:

(1) Plantes se procurant la nourriture organique par leurs propres moyens, c'est-à-dire plantes à chlorophylle.

(2) Wisconsin Geol. and Nat. Hist. Survey, Bulletin no 64, Sér. no 13, 1922, p. 150 . 
I. - Les Chlorophycées ou Algues vertes, dépourvues de pigments surnuméraire, dont les chloroplastes colorés en vert sont formés des mêmes pirments que chez les végétaux supérieurs : chlorophỵlle, carotène, xantophylle.

II. - Les Phéophycées ou Algues brunes; leurs chloroplastes contiennent non seulement les mêmes pigments que chez les Chlorophycées, mais encore un pigment caroténoüde brun : la phycoxanthine. Pendant la vie de la plante, ce pigment masque les précédents, mais il disparait au moment de sa mort. Les Diatomées, en raison de leurs plastes bruns, sont rapprochées des Phéophycées.

III. - Les Rhodophycées ou Algues rouges, chez lesquelles aux pigments ordinaires est mèlé un pigment surnuméraire de teinte rouge : la phycoérythrine, qui masque la chlorophỵlle. Ce piyment surnuméraire n est fixé aux chloroplastes que pendant la vie ; lorsque l'Algue meurt, il diffuse dans l'eau.

Enfin, malgré I'absence de plastes et de noyau différencié, est également rattaché aux Algues, le groupe des Cyanophycées ou Algues bleues. Le cytoplasme de ces Algues contient, à l'état diffus, de la chlorophylle associée à un pigment bleu : la phycocyanine, qui masque plus ou moins la première.

\section{II. - istribution GÉograpmoue et dissémination}

La distribution géographique des Algues d'eau douce, exception faite des $\Lambda$ lgues aériennes qui paraissent plus spécialement localisées aux régions tropicales humides, constitue, sauf quelques variantes dues aux climats, des formations végétales entièrement semblables dans toules les zones du globe. Ce pouvoir de dispersion dépend beaucoup plus de leur grande faculté d'adaptation aux conditions physico-chimiques variées du milieu (ubiquisme) (1), que de l'influence du lieu géographique.

Ce cosmopolitisme des Algues est également le résultat de leur facile transport par les principaux agents disséminateurs; l'eau, comme élément essentiel a leur existence, est le plus important de tous : des montagnes aux plaines les Algues sont cmportées par les ruisseaux jusqu'aux rivières et aux fleuves, peuplant dans ces trajets les étangrs et les lacs. Les oiseaux aquatiques, en particulier les migrateurs, sont également les meilleurs auxiliaires après l'eau; ils favorisent leur dispersion au loin en emportant la vase qui recèle leurs germes. Enfin, le vent lui-mìme est un agrent de transport des hypnospores (organes de conservation de ces végétaux) doués d'un certain pouvoir de résistance à la dessication.

(1) Ubiquistes : plantes qui peuvent vivre dans des stations diverses. 
Alternance du cycle végétatif.

Par la rapidité des diverses phases de leur manifestation vitale, les Algues d'eau douce son remarquablement résistantes aux plus excessives variations du milieu ; elles savent utiliser au maximum, en un minimum de temps, les conditions favorables à lcur activité végétative. La plupart ont rarement la mème abondance pendant toute la période annuelle ; beaucoup passent, au cours des diverses conditions saisonnières, par des états de vie ralentie où elles semblent disparaître ; dans ce stade quiescent, elles assurent leur conservation au moyen d'orranes spéciaux : hypnospores végétatives ou sexuelles (ocufs) qui s enfoncent dans la vase. Lorsque les conditions biologiques utiles réapparaissent, il se produit au cours des diverses saisons, une prédominance d'Algues caracléristiques des habitats peuplés par ces végétaux.

Les habitats et leurs formes algales caractéristiques.

Depuis leur vie entièrement sulmeráce, point de dípart de leur évolution ancestrale, jusqu'à la vie aérienne qui en représente l'ultime deg̨ré, les Algues d'eau douce occupent, en raison de leur grand pouvoir d'adaptation, les milieux les plus différents. Certaines même habitent dans le sol et y mènent une vie saprophỵtique; d'autres se développent à l'intérieur des plantes ou des animaux aquatiques.

Leur répartition écologique est un des plus intéressants problèmes de l'Algologie ; aussi croyons-nous utile de donner par le tablcau qui suit, un aperçu des multiples stations qu'elles occupent dans la nature.

\section{1. - Algues vivant au sein des eaux douces de nombreux habitals.}

A. - Dans les eaux dormantes ou à lrès faible courant : Limnophiles :

$1^{\circ} \mathrm{Ni}$ incrustantes, ni perforantes :

a) Fixécs sur un support quelconque, d'une façon plus ou moinspermanente : Benthos :

a. Sur le sol ou la vase submergée : Géophiles ou Limicoles :

(Tetraspora gelatinosa (Vauch) Desv.; bon nombre d'Algues filamenteuses an début de leur développement : Spirogyra, Cladophora, Stigcoclonium, etc...).

$\beta$. Sur les pierres submergées : Lithophiles :

(Coleochaete, Ulothrix, Cladophora, Oedogonium, etc...).

$\gamma$. Sur les végétaux supérieurs submergés : Phylophiles :

(Stigeoclonium, Draparnaldia, Microlhamnion Kützingianum

NaEc., Chaetophora, Coleochaete, etc...).

b) Vivant à l'intérieur des végétaux ou des animaux aquatiques :

a. Dans les végétaux : Endophytes :

(Endoderma Cladophorae Horxby à l'intéricur des Cladophora; 
Chlorochytrium Lemnae CoHs., dans les Lemna; Anabaena Azollae STrasb., dans'les Azolla; etc...).

B. Dans les animaux : Endozoïques:

(Les gonodies trouvées à l'intérieur des Hỵlres d'eau douce; dans les Protozoaires Ciliés, Paramecium, Ophrydium; dans les Epongres d'eau douce et les Mollusques, sont dues aux espèces du genre Chlorella).

$2^{\circ}$ Incrustantes, perforantes ou cariantes :

a. Dans les eaux froides - Algues dont les filaments sont entourés d'une gaine plus ou moins épaisse formée par un dépôt de carbonate de chaux : Incrustantes:

(Congrosira Scourficldii WEEsT, Chlorotylium calaractum Küzz., Calolhrix parietina (NaEc.) Tnun, Rivularia haemalites (DC.) AG., Oncobyra rivularis KüTz., etc...).

B. Perforantes du test des Anodontes : Perforantes :

(Foreliella perforans Chop. sur les coquilles des Anodontes).

$\gamma$. Perforantes par carie progressive des pierres et des coquillagres: Calcivores :

(Gongrosira codiolifera Chod., Hyella fonticola Hubert el Jadiv, Schizothrix fasciculata (NAEG.) Gom., etc...).

aa) A un autre point de vue, on distingue les Algues unicellulaircs ou pluricellulaires douées de motilité, ou passives et flottantes au gré des vagues formant le Limnoplancton:

a. Flore planctonique des grands lacs, ayant au moins 30 mètres de profondeur : Pélagoplancton :

(Sphaerocystis Schroteri CHod., Botryococcus Branii KüTz., Oocystis lacustris CHoD., Dactylococcus lacnstris ChoD., Gomphospharia lacustris (inop,, Cyclolella Bodanica EuLst., etc., etc...).

ค. Idem des petits lacs ou lacs - étangs de faible prolondeur : Limnoplancton proprement dit :

(Les Pediastrum, Coliastrum, Pandorina morum (Mür.l.) Bony ; les Oocystis, Lagerheimia ; beaucoup de Desmidliées : Closterium, Cosmarium, Staurastrum ; des Mallomonas et Dinobryon; beaucoup de Diatomées (en particulier comme caracléristiques les genres (yclotella, Rhizosolenia) ; les Peridinium el Ceratium; des Cyanophycées comme Gomphosphaeria, Microcystis et autres genres à formes planctoniques, etc.).

$\gamma$. Idem des étangs et mares de très faible profondeur envahis ou non par les Macrophytes : Héléoplancton:

(Chlamydomonas, Gonium et autres Volvocales; nombreuses 
Chlorococcales, Ulothrichales, Conjugatées, Desmidiées; Botryococcus Braunii, Ophiocylium; nombreuses Chrysophycées, Diatomées, Péridiniées, Cyanophycées).

$\delta$. Idem des étangs tourbeux et tourbières à Sphaignes : Sphagnoplancton.

(Schizochlamys delicatula West, Eremosphara viridis DE Bary, Oocystis plur. sp., Geminella mutabilis (Bréb.J Wille, Spirotonia plur. sp., également Cylindrocystis et Netrium; un grand nombre de Desmidiées avec les grandes formes caractéristiques des Micrasterias et Euastrum ; Chlorobotrys ' regularis (West) BoHıN, nombreuses Diatomées, en particulier Eunolia, Frustulia, Pinnularia, etc...; également beaucoup de Cyanophycées).

B. - Dans les eaux counantes des rivières et des fleuves : Potamophiles :

a. Fixées plus ou moins temporairement-sur les Hydrophytes des diverses zones ou sur les matériaux des berges : Formes-benthiques ou littorales:

(Ulothrix, Hormidium, Microspona, Chatomorpha, Cladophora, Stigeoclonium, Chatophora; nombreuses Diatomées; Chantransia, Batrachospermum, Lemanea, Hildcbrandtia, Xenococcus ainsi que beaucoup d'autres Cyanophycées, etc...).

$\beta$. Libres : pélagiques ou passives : Potamoplancton :

- Beaucoup d'espèces euplanctoniques appartenant au Limnoplancton se retrouvent ici ; un grand nombre des formes littorales ou benthiques facultatives peuvent y figurer également; le Potamoplancton est surtout un Plancton d'emprunt. Cependant les formes suivantes paraissent plus ou moins caractéristiques de cet habitat : Cyclotella glomerata Bachmass, Ceratoneis arcus (Enn.) Kïrz., Nitzschia actinastroides (Lемм.) Vav Goon, Asterionella gracillima (Hantz.) Ilem., Fragilaria crotonensis (EDw.) Kiтt., Synedra acus plur. var., Synura uvella EнH., Actinastrum Hanlzschii LAGERH., etc...

II. - Algues vivant au dehors des habitats précités, mais seulement dans des biotopes aqueux, très aérés, ou sur des supports aériens divers mais soumis à un régime hygroscopique variable :

A. - Sur des surfaces suintantes de positions variées, dans de petites collections d'eau, sur des supports imbibés : Formes subaeriennes ou aérophiles :

a. Sur les parois des rochers suintants :

$\Delta$. A suintements permanents : Mésophiles : - Pediastrum integrum NAEg., Oocystis plur. sp., Glaucocystis Nostochinearum 
Itzıcs., Mosolaenium plur. sp., Gonatozygon Brebissonii DE Bary, Closterium plur, sp., Cosmarium plur. sp., Staurastrum Meriani Rerssch. ; des cystes de Chrysomonadinées; de nombreuses Diatomées ; Aphanocapsa Grevillei (HAss.), RAвн., A pulchrá (Küтz.) RAвH., des Lyngbya et Oscillatoria, etc...

$\Delta \Delta$. A suintements temporaires: Xérotiques: - (Hormidium dissectum Chod., Cylindrocystis crassa DE BARY, Achnanthes coarctata Breb., Melosira Rosseana Raill., Pinnularia borealis Eнн. ; nombreuses Cyanophycées des genres Gleocapsa, Chroococcus, Phormidium, Symploca, Hassalia, Sligonema, etc., etc...

§. Dans les coussinets de Mousses imbibées d'eau : Bryophiles :

La plupart des espèces de l'habitat précédent, en plus quelques Diatomées plus fréquentes ici, particulièrement en montagne : tels que Tetracyclus rupestris (A. Bn.) Grun., Diatomella Balfouriana Grev., qui sont toutes deux des formes alpines, enfin des Cyanophycées comme Aphanocapsa, etc...

$\gamma$. Dans les sources, hassins des fontaines, les cuvettes des chutes d'eau et des cascades: Crénophiles :

Ulolhrix zonata (Wen. et MoHR), Küтz., Hormidium rivulare Küтz., Microspora amcna (Küтz.) LAGERH., Chøtomorpha, Clodophora; un grand nombre de Diatomées caractéristiques des Chantransia et Batrachospermum, etc...

B. - Algrtes qui accomplissent leurs fonctions vitales hors de l'élément aqueux, mais seulement au dépens de l'eau météorique, et qui, de ce fait, supportent sans dommage les effets dune dessication plus ou moins prolongée : Formes aériennes :

a. Sur la terre : Terricoles épigées :

Mesotaenium chlamydosporum D: B.ru, Cylindrocystis Brebissonii, Zygnema ericetorum (KüTz.) HaxsG., Chlorococcum humicola (NAG.) RaвH., Hormidium flaccidum A. Br., Prasiola crispa (Lighte) MinEtiH., OEdocladium protonema Stahl., Rhizoclonium riparium ILAhv., Vaucheria hamata Walz, V. repens Hass,, Symploca muralis Kütz.

$\beta$. Sur la terre ou sur les murs souillés : Urophiles :

Schizogonium murale KüTz., Porphyridiam cruentum (AlF.), NAEG.

$\gamma$. Sur le limbe des feuilles: Epiphylles:

Trentepohlia, Cephaleuros, Phycopeltis, Phyllosiphon, Scyloncma Hofmanni AGardr, etc...

\&. Sur l'épiderme ou les poils des animaux : Epizoiques :

Dermatophyton, Trichophilus. 
. Sur les rochers, les pierres et les murs : Saxicoles:

Trentepohlia aurea (L.) Mant., T. Jolithus (L.) Wallr., Prusiola leprosa Kürtz.

甲. Sur le chapeau de certaines Polyporacées: Ligni-fongicolcs :

Chlamydomonas fungicola De Puymay, Chlorella ellipsoidea Grineck, Keralococcus caudatus Pisch., Hormidium dissectum Cirop., H. pseudostichococcus HeEning, Stichococcus baccilaris NAEG.

$\pi$. Sur les troncs lisses ou les écorces des arbres : Corticoles :

Trebouxia arboricola Puym., Iormidiopsis crenulata (Kütz), Herhivg., Prolococcus Nacgelii Chob., Pleurastrum lobatum (Chod, Printz, Trentepohlia abietina (Flotow) Hansa., T. odorata (Wiggens) Wittr...

ө. Sur les toits de chaume: Calamicoles :

Avec des Diatomées et des Cyanophycées se trouvent les cspèces suivantes : Mesotacnium macrococcum (KüTz.) RoY et Błsset, Pleurococcus vulgaris MeneGil., P. Naegelii Crrod, Dactylococcus bicaudatus A. Bн, ainsi que certaines espèces des habitats précédents.

III. - Algues vivant sur la neige cl la glace des régions froides et des hautes montagnes : Cryoplancton :

Chlamydomonas nivalis WiLle, Encylonema Nordenskiceldii Berga., Raphidium nivale Chod., R. Víreti C.нod., Scotiella nivalis (Chod.) Fratscir.

1V. - llgucs vivant dars les bassins des sounces chaudes et des geysers : Thermophiles :-

Certaines formes eurythermes de Diatomées el Cyanophycées.

Y. - Flore algale souterraine du sol : Terricoles hypogées :

Chlorococcum humicolum (NAEG.J RABH., Trochiscia aspera ReInscir) Hansg., Ulothrix subtilis KüTz. var. variabilis (KüTz). KırchN., Bumilleria exilis KLEıs., Navicula mulica Kütz., N. atomus (NAEg.) Gruv., Hanlzschia amphioxys (Eur.) Grun., Plectonema Battersii Gom,, Phormidium plur. sp.

\section{Les peuplements utiles.}

De tous les nombreux peuplements en Algues, figurant dans ce tableau, nous n'étudierons ici que ceux qui jouent un rôle utile dans la nourriture des Poissons, soit indirectement par interposition de la faune microscopique leur servant de proie, ou encore directement dans leur alimentation. Toutes les associations algales nourricières appartiennent uniquement aux 
formations benthiques et planctoniques de la première partie de notre tableau ; la composition de leurs éléments foristiques est plus ou moins caractéristiques des divers habitats, ainsi que des conditions écologiques qui régissent ces derniers.

Les deux grands modes d'existence que représentent le Benthos ou le Plancton, entraînent chez les 1 lgues deau douce un certain nombre de dispositifs bjologiques particuliers : moyens de fixations pour les Algues benthiques, mécanismes de flottaison pour les Algues planctoniques. La différenciation d'un élément quelconque de l'organisme algal, ou encore la spécialisation morphologique totale te celui-ci, à l'un de ces deux modes d'existence, déterminent de nombreux exemples de convergence de forme chez des espèces de position systématique parfois fort éloignée.

\section{III. - LE BENTHOS}

Ghez les Algues benthiques ou littorales, indifférentes à la nature du support sur lequel elles vivent fixées, habitant par conséquent de nombreuses formations, les divers moyens de fixation de leur thalle peuvent se résumer, pour les Euchlorophycées en particulier, par les trois types biologriques suivants :

I. - Type en écusson ou en rhizome à symétrie dorsiventrale.

II. - Type characioüde, permanent, ou seulement au stade juvénile.

III. - Type Hormotila à support gélifié, simple ou ramifié.

Dans le premier type à différencialion dorsiventrale, par exemple le Colcochacte scutala Briér. (Fig. 1, pl. V), la thalle discoïde, provenant d'une ou plusieurs cellules centrales se ramifiant en filaments dichotomiques radiés, est parsemé à sa périphérie de poils murueux, tuniqués, tous dirigés dans le milieu liquide, alors que les cellules pseudo-parenchimateuses forment chez cette Algue un coussinet étroitement fixé sur le substratum. Chez 1 phanochneter repens $\mathrm{A}$. Br. (Fig. 2, pl. V), ce dispositif se réduit à l'élat de filaments pleurococcoïles rampants, non agrégés en thalle discoüde, à surface dorsale surmontéc de longs poils renflés à leur base. Enfin, ce somatropisme (1) particulier se manifeste éralement chez les Batrachospermum avec un thalle rampant rhizomateux, ou encore en forme de talon chez Stigcoclonium. Ainsi, par l'absence de tout géotropisme (2) et les effets du somatropisme, toutes les $\Lambda$ lowes pourvues d'un de ces moyens de fixation peuvent se développer dans n'importe quelle orientation sur les organes des végétaux submergés.

Avec les Algues du type characioïde à régime benthique permanent, figurent également des Algues libres, qui, au début de leur développement,

(1) Influence directe du substratum sur la croissance des organismes.

(2) Action de la pesanteur sur la direction des organes des plantes. 
vivent fixées sur un support au moyen du même dispostif, par exemple chez OEdogonium plur. sp. (Fig. 4, pl. V), ou encore les Microthamnion. Dans leur ensemble, toutes ces Algues ont pour caractère commun de donner naissance lors de la germination de leurs zoospores ou ceufs, à des plantules characioïdes pourvues d'un crampon en forme de disque ou de griffe, sur. montées en un stipe plus ou moins développé se terminant par le thalle filamenteux ou vésiculeux de l'Algue. Chez les petites Algues unicellulaires comme es Characium (Fig. 3, pl. V), qui offrent peu de prise au mouvement de l'eau, l'appareil de fixation se réduit en un cordon cellulosique plus ou moins allongé, s'appliquant au substratum par un épaississement basilaire discoïde. Ce dispositif est tout autre pour les Algues comme les Draparnaldia, dont la thalle pluricellulaire, filamenteux ou arbusculeux, présente une plus grande surface au choc des vagues : il se forme à la germination un rhizome, entouré de rhizoïdes adventifs issus des premières cellules du thalle. Dans ces diverses réalisations du type characiö̈de, la structure générale ést radiaire avec différenciation en base et sommet.

Enfin chez les Algues benthiques du type Hormolila, tel que Mischococcus confervicola NaEg. (Fig. 5, pl. V), appartenant aux Xanthophycées, la zygospore ou la zoospore, fixées au moyen d'une substance adhésive, rompent à la germination leur enveloppe pour se diviser en deux plans, dont un forme une sorte de colonne tubuleuse gélifiée, restant adhérente au support et qui soulève la cellule issue du second plan ; cette dernière qui reste également fixée sur la précédente, donne naissance à un dispositif semblable. Par le développement successif d'autres cellules, cette Algue donne naissance à un thalle ramifié en forme d'arbuscule, vivant en épiphyte sur de nombreuses Algues filamenfeuses.

Les divers types de fixation que nous venons de passer en revue chez les Euchlorophycées, se retrouvent plus ou moins modifiés ou dégradés chez les formes benthiques des autres classes d'Algues, par exemple pour les Rhodophycées: Lemanea, Batrachospermum et Chantransia. De même les Chrysophycées épiphyles présentent, avec des formes palmellö̈des (1) attachées, des formes characiö̈des comme Phaeothamnion confervicolum LAGERH.

Quant aux Diatomées, extrèmement abondantes et varíes dans les eaux douces, d'une façon généralc les formes benthirques ou littorales sont fixées à leur support au moyen d'une substance muqueuse secrétée par la cellule. Dans plusieurs genres de Diatomées coloniales, cette substance est nettement localisée ; par exemple les frustules (2) des Diatoma et Tabellaria, disposés en ziğ-zag, sont reliés entre eux par un ciment muqueux secrété à un

(1) Formes palmelloides : - Algues dont les cellules sont associées en colonies gelatineuses, chaque cellule étant un individu.

(2) Frustule : - carapace siliceuse formant l'enveloppe au squelette des Diatomées. 


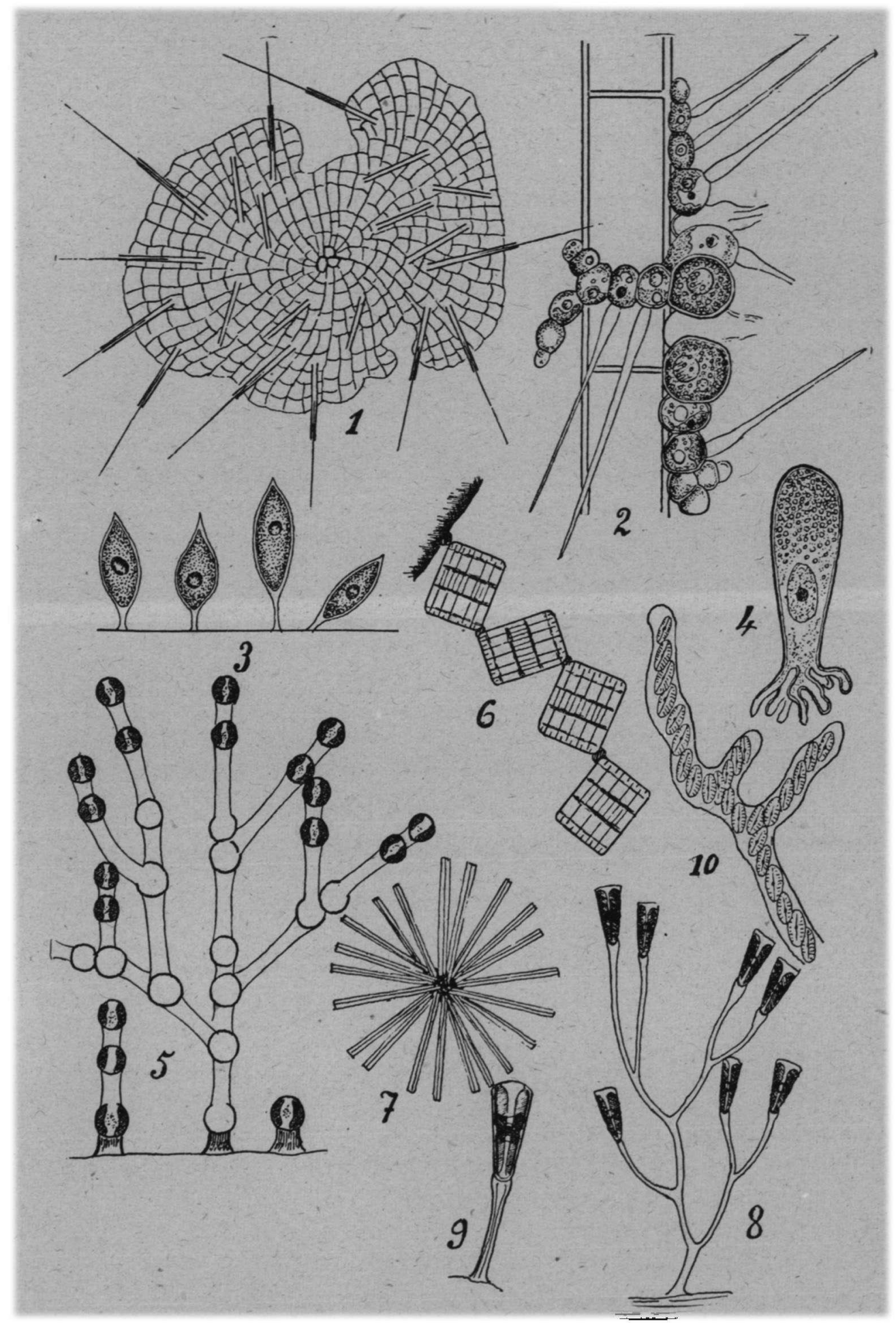

Planch z V. - Modes de fixation chez les Algues benthiques.

1, type dorsiventral Coleochaete scutata a thalle pseudo-parenchymateux; -2 , ehez Aphanochaete repens à fllaments pleurococcoïdes; - 3 , type characioïde unicellulaire chez Characium Pringsheimii A. Br. ; - 4, à jeune plantule characioïde chez un Odogonium sp.; - 5, type hormotila $M$ ischococcus confervicola; Chez les Diatomées : - 6, frustules rèunis en zig-zag par des coussinets muqueux chez Tabellaria /locculosa; - 7 , en touffe chez Synedra ulna; - 8, en stipe gélatineux ramifié chez un Gomphonema sp; - 9, sur pédoncule gélatineux chez Gomphonema constrictum; - 10, à l'intérieur d'un tube gélatineux ramifié chez Cymbella caespitosa. 
des angles de la cellule, le frustule basal étant lui-même fixé au substratum par un semblable coussinet (Fig. (6, pl. V). Beaucoup de Diatomées pennées exudent ce mucus par un pore situé ordinairement au voisinage d'un ou des deux pôles de la valve; il forme généralement une saillie très nette sur la membrane siliceuse de la valve.

Il convient de ranger les Diatomées fixées en deux catégories, selon qu'elles vivent agrégées en colonies flottantes, semi-planctoniques, attachées temporairement en un point à leur support, ou bien qu'elles apparaissent hałituellement en épiphytes, fixées isolément,. ou en un groupe à leur support.

Dans la première catégoric, les colonies sont figurées par des individus réunis les uns aux autres en chaines ou en filaments (certains Melosira), ou en zigr-zag (Dialoma et Tabcllaria), enfin en rubans (Fragilaria). Ce sont des formes planctoniques facultatives, adaptées à la vie flottante.

La deuxième catégorie, la plus nombreuse en représentants, se résume par les divers types suivants :

a) Frustules rarement isolés, le plus souvent rassemblés en touffes, fixés au substratum par un coussinet muqueux : beaucoup de Synedra (Fig. 7, pl. V), Nitschia, également certaines espèces à pédoncule gélatineux très court comme chez plusieurs Comphonema (Fig. 9, pl. V).

b) Frustules insérés directement sur leur substratum par une des faces valvaires, tels que les Epithemia et $A$ mphora, dont la face ventrale valvaire avec ses.deux raphés est appliquée sur le support; également le Cocconeis pediculus, à valves fortement arcquées, s insère par la valve pourvue de raphés.

c) Frustules en robés dans une pellicule gélatineuse tapissant la vase, les pierres ou tous autres supports submergés: un certain nombre de Navicula et de Nitzschia.

d) Frustules contenus dans des tubes muqueux. Pour beaucoup d'espèces ce mode de vie est facultatif. Dans ce dispositif, un grand nombre d'individus sont inclus dans un tube gélatineux commun, simple ou ramifié : Frustulia, Navicula, Cymbella sect. Encyonema (Fig. 10, pl. V), Nitzschia sect. Homococladia, etc. Dans certains cas, l'on a observé la coexistence de deux espèces dans le même tube.

e) Frustules pédonculés. Ce mode de fixation, le plus fréquent, est également facultatif pour beaucoup d'espèces. Ici les individus sont fixés par lour extrémilé à une des ramifications hyaline du stipe gélatineux attaché au support, ou encore simplement iusérés à un simple pédoncúle de mème nature. Ce mode de liaison est représenté chez la plupart des Gomphonema (Fig. 8, pl. V) ; de même pour un certain nombre de Cymbella sect. Cocconema, les Rhoicosphenia et Achnanthes plur. sp. 
Enfin, après les Diatomées, il faut réserver une place à part aux quelques Algrues qui habitent le mucus des Batrachospermum, Coleochaete, ou les gelées tégumentaires de certaines Cyanophycées; ce sont plus particulièrement les Chaetonema et Gloeocystis. Il est probable que ces Algues gélophiles ne sont pas sans joucr un rôle utile dans le développement des Alevins qui recherchent cet habitat.

\section{iv. - LE PLANCTON}

La signification du terme Plancton, employé pour la première fois en 1887 par le Suédois Hersex, exprime l'ensemble de tous les êtres, végétaux et animaux microscopiques, qui flottent ou rui nagent passivement dans l'eau. Si cette définition première du Plancton n'implique aucune confusion quant áux organismes marins, l'étendue et la composition de ce milieu étant parfaitement définies, il n'en est plus de mème lorsque celle-ci sapplique aux formations d'eau douce. Ici la grande diversité des milieux et des facteurs édaphiques rendent cette notion beaucoup plus confuse; aussi les Hydrobiologistes ont-ils élé amenés à créer, parfois même avec exagération, nombres de termes pour définir d'une manière précise la spécificité d'un Plancton d'eau douce.

Les éléments figurés communs au Plancton marin comme à celui d eau douce sont les suivants : le Plancton végétal ou Phytoplancton, le Plancton animal ou Zooplancion, enfin le Tripton, formé par les particules organiques et minérales en suspension ou flottant dans l'eau, qui constitue le Planctotripton. L'ensemble de ces trois éléments, vivants et inanimés, c'est-à-dire tout ce qui reste sur un tamis après fillation de l'eau, porte le nom de Seston.

\section{Le Phytoplancton.}

Le rôle capital des Algues dans la Biologie du monde aquatique et, en tout premier lieu, dévolu aux espèces planctoniques; nous avons indiqué précédemment, d'une façon générale, la grande importance de ces microphytes comme créateurs de substances nutritives indispensabes à l'entretien de la vie animale. Erralement nous avons énuméré les nombreux Planctons végétaux selon leur origine stationnelle.

Quant à la répartition el à l'évolution du Phytoplancton, ces deux points essentiels de la vie des $A$ lgues, ils sont en rapport avec les multiples effets des facteurs physico-chimiques et biotiques du milieu; ces facteurs sont éralement pour la plupart variables en fonction de lespace et du temps. L'étude des nombreux problèmes qute posent toutes ces données appartient au domaine de l'Hydrobiologie (1).

Il importe seulement ici, pour l'exposé du sujet que nous nous sommes

(1) Voir P. Vivier. - Les Principes de la Limnologie. - Bulletin Français de Pisciculture, - nos 68, 69, 70 et 71, Février-Mai 1934. 
fixé, de développer quelques notions précises sur les moyens mis en ocuvre par les Algues dans leur adaptation à la vie planctonique, ainsi que les diverses théories du mécanisme de la flottaison chez ces végétaux inférieurs.

Tout d'ahord, quelques explications sur l'origine intrinsèque du Phyloplancton. En général, les organismes algales de cette grande formation passent au cours de leur vie par deux phases distinctes : $-1^{\circ}$ une phasé active ou cinélique, celle de la vie planctonique $-2^{\circ}$ une phase inactive, correspondant à la phase immobile ou acinétique, représentée par la production d'organes de conservation, tels que les cystes, hypnospores el akinètes, dépourvus de motilité et de moyen de flottaison, et qui tombent au fond de lieau.

Cette alternance du cycle végétatif des Algues planctoniques se traduit par des périodes d'apparition et de disparition totale ou partielle de cellesci. Ces phénomènes ne signifient paas toujours qu'un ou plusieurs éléments figurés d'un Plancton prennent naissance dans le milieu même où ils sont observés, c'est-ả-dire quiils soient endogénéliques; au contraire, il peut arriver, ce qui est fréquemment le cas des éléments du Potamoplancton, que l'une ou l'autre phase, parfois les deux, prennent naissance en des lieux éloignés; ces éléments sont alors d'origine externe ou allogénétiquc.

Avant de traiter de la forme comme élément essentiel de la flottaison, nous dirons seulement quelques mots sur la grosseur des Planctons et son importance utilitaire. La valeur nutritive des Algues du Phytoplancton, en particulier celles des espèces unicellulaires qui constituent la nourriture de choix des Cladocères phytophages, dépend en premier lieu de leur grosseur, autrement dit de leur facilité d'absorption par les ravisseurs animaux. Cet important caractère est exprimé par la classification suivante des Planctons :

Mieroplancton. - De $20 \mu$ jusqu à quelques millimètres, représenté par la majorité des espèces planctoniques uni et pluricellulaires. y compris les formes coloniales.

Nanoplancton. - Inférieurs à $20 \mu$; beaucoup de ces espèces passent à travers les mailles du filet fin; elles constituent le Plancton de centrifugation.

Lltraplancton. - Inférieurs à $5 \mu ; \mathrm{y}$ figurent surtout les Bactéries. $L$ étude de ce Plancton se fait par filtration sur bougie ou culture sur plaque.

Malgré l'état élémentaire de nos connaissances actuelles sur la sélection de la faune aquatique à l'égard des microphytes utiles a sa nutrition, l'on peut admettre avec certitude le rỏle prépondérant du Fanoplancton comme élément nourricier des petits animaux aquatiques, en particulier chez les Crustacés inférieurs (Entomostracés). 


\section{Les formes typiques chez les Algues planctoniques.}

Les nombreuses formes d'Algues unicellulaires, isolées ou groupées en colonies du Plancton d'eau douce, se ramènent aux trois types morphologiques suivants : les formes allongées, plates, sphériques ou cubiques (isodiamétriques), selon l'accentuation de leur développement dans une ou plusieurs des trois dimensions.

La mème variété de forme chez les Algues planctoniques se retrouve, à peü d'exceptions près, chez les Algues non planctoniques. La convergence morphologique ne laisse apercevoir aucune conclusion positive sur le mode de vie particulier. Cépendant le caractère de la forme n'est pas moins de première importance chez les Algues planctoniques, surtout si l'on considère les nombreuses combinaisons qui résultent des trois types fondamentaux : soit séparément ou réunis entre eux, ou encore combinés à des éléments secondaires tels que les formations gélatifieuses, la production d'appendices, la préscuce de corps gras on de vacuoles gazeuses. Tous ces éléments représentent autant de moyens supplémentaires s'ajoutant à la forme, augmentant ainsi le pouvoir de flottaison de ces microphytes.

\section{Formes à une dimension (P. VI).}

Ici l'accentuation du développement de la forme est donnée dans unc seule direction ; ce type est représenté par des Algues unicellulaires isolées ou associées en filaments simples, ou groupées de diverses façons.

\section{Cellules isolées :}

$1^{\circ}$ Forme en baguette, commune chez les organismes du Phytoplancton, clle peut etre prismatique ou cylindrique et fréquemment arrondie aux extrémités : Synedra (f. 1), Nitzschia (f. 2), Gonatozygon monotaennium (P. 3).

$2^{\circ}$ Forme en aiguille, semblable à la précédente, mais à extrémités pointues : droite Synedra (f. 1), Nitzschia (f. 5), Closterium acutum (f. 6), courbée Ankistrodesmus longissima (f. 7 ), cn spirale Ankistrodesmus spiralis (f. 8). Chez certaines Algues, les extrémités sont très allongées, alors que la partie médiane est cylindrique ou fusiforme : Rhizosolenia (f. 9), Ankistrodesmus setigera (f. 10).

Cellules associées en colonies:

$3^{\circ}$ Forme en filament; très fréquente dass le Plancton, mais non exclusive; elle est constituće de cellules associées les unes aux autres par leurs extrémités; elle est droite ou plus ou moins courbée, sinueuse, spiralée : Oscillatoria (f. 11), Lyngbya (f. 12), Phormidium (f. 13), Aphanizomenon (f. 14), Anabaena (f. 15), Melosira (f. 16), quelques espèces de Cyclotella f. 17), Tribonema (f. 18), Mougeotia (f. 19). Les cellules des filaments peuvent ètre de forme discoïde, cylindrique, sphérique, mais comme elles ne sont que des unités, seuls leur association en filament représente une formation à une dimension.

$4^{\circ}$ Forme en touffe, celle-ci se présente lorsque les cellules en baguettes 


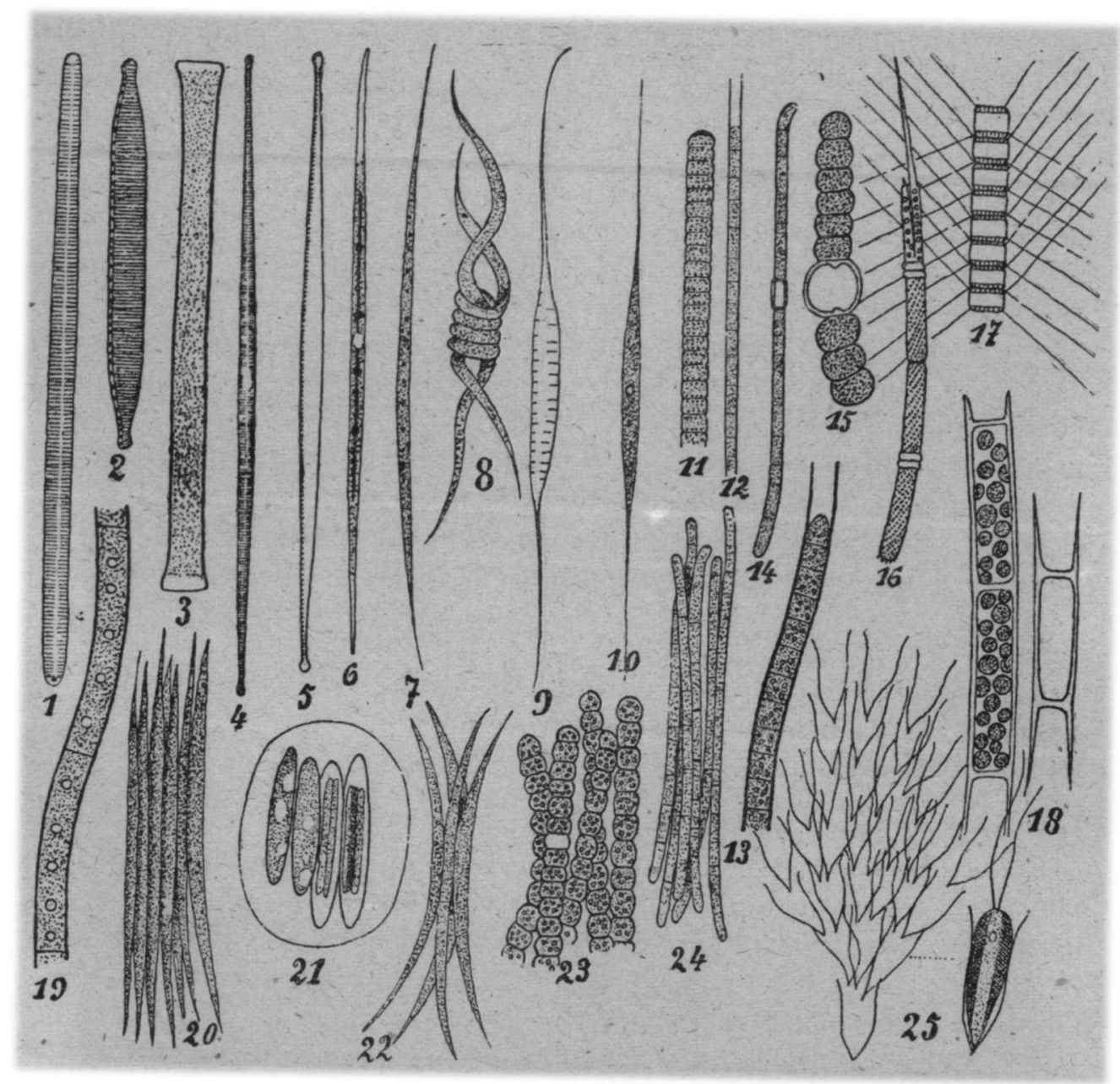

Pr.anche Vi. - Formes d'Algues à une dimension.

A. - Type baguetre : - figs. 1 Synedra ulna (Nitzsch) Fhr., var acqualis (Kütz.) Hust.; - 2 Nitzschia palea (Kïtz.) W. Sm. ; - 3 fonatozygon monotacnium De Bary.

B. - Type alquil.e: : - figs. 4 Synedra acus Kütz., var. radiuns (Kütz.) Hust.; - 5 Nitzschia gracilis Hantz.; - 6 Closterium acutum Bréb.; - 7 Ankistrodesmus longissimus (Lemm.) Wille ; -8 A. spiralis (Turner) Lemm.; - 9 Rhizosolenia longiseta Zach.; - 10 Ankistrodesmus setigerus (Schroder) G. S. West.

C. - TyPe Fli.Ament : - figs. 11 Oscillatoria tenuts Ag.; - 12 Lyngbya limnetica IJemn. ; 13 Phormidium papyraceum (Ag.) Gom.; -11 Aphanizomenon flos-aquae (L.) Lalfs; - 15 Anabaena planctonica Brunth.; - 16 Melosira granulata (Ehr.) Ralfs, var. angustissima Müll. ; - 17 Cyclotella chaetoceras Lemm. ; - 18 Tribonema bombycinum Derh. et Sol. ; - 19 Mougeotia viridis (Kütz.) Wittr.

D. - TYPE тоurFe : - figs. 20 Ankistrodesmus biplex Reinsch; - 21 A. Pfitzeri (Schrogder) G.S. West; - 22 A. falcatus (Corda) Ralts; - 23 Trichodesmium lacustre Kleb; -24 Aphanizomenon flos-aquae (L.) Ralfs; - 25 Dinobryon Sertularia Ehr. 
ou en aiguilles se réunissent longitudinalement sur un même point de leur hauteur : Anlistrodesmus biplex (f. 20), A. Pfitzeri (f. 21), A. falcatus (f. 22). Dans celte mème calégrorie figrurent des Algues dont les filaments séparés s'unissent également en touffe : Trichodesmium (Oscillatoria) lacusthe (f. 23), Aphanizomenon flos-aquate (f. 24). Tout autre est la forme en touffe chez les Dinobryon qui sont des Algues essentiellement planctoniques ; ici les éléments de la colonie (cellules en forme de capsule (f. 25) ne sont pas toutes à la mème hauteur, mais placées par étages où leur nombre augmente rérrulièrement depuis la première cellule de base jusqu'au sommet de la colonie; l'ensemble pourrait être considéré comme une forme à deux dimensions.

Lorsque les cellules en forme de baguette sont rangées horizontalement malg̣ré parfois uñ assemblag̣e plus ou moins làche, les colonies qui en résultent sont en forme d'ćtoile, de chaine ou de cordon se rapprochant des formes à deux dimensions avec lesquelles on les range. Ces mêmes cellules en baguettes peuvent donner éralement des colonies disposées en sphères (Actinastrum, Nitzschia holsatica), appartenant aux formes à trois dimensions.

\section{Formes à deux dimensions (Pl. VII).}

Les formes dont le développement est accentué dans deux directions spaciales, sont très abondantes dans le Phytoplancton; leur surface et leur faible épaisseur offrent Jes qualités idéales pour la flottaison. Ces surfaces sont peu stables et par celin mème en perpéfuelle agitation sous l'action du mouvement de l'eau, ce qui a pour conséquence de ralentir leur chute dans ce milieu. La stabilité de ces formés, en particulier dans leur horizontalité, est accrue par des dispositifs spéciaux : rangres d'airuillons sur leur surface, dentelures ou échancrures sur les lords de cefle-ci.

Les formes à deux dimensions sont égralement représentées par des Algues unicellulaires isolées, ou associécs en colonies.

Cellules isolées :

$1^{\circ}$ Forme en disque ou en médailhe, fréquente chea les Diatomées à symétrie rayonnante (centriques) : Cyclotella (f. 26), Stephanodiscus (f. 27).

$2^{\circ}$ Forme lenticulaire, plus rare que la précédente : Phacotus lenlicularis (f. 28).

D'autres formes du mème type sont également fréquentes, plus spécialement chez les Diatomées : - ovales Surirellia (f. 29), Cymatopleura (f. 30), - elliptiques : Wheya (f. 31), Rhizosolnia (f. 32), - en forme de semelle : Cymalopleura solea (f. 33).

Il exište de mème des formes plates anguleuses : simples chcz eertains Teträ̈dron (f. 34), on combinées à des accessioires secondaires tels que des piquants, ou à des appendices angulaires : Pseudotelraëdron (f. 35).

Cellules associées en colonies :

Les divers modes d'aggrégation cellulaire en surface donnent naissance aux formes coloniales suivantes : 
$1^{\circ}$ La forme en disque pluricellulaire d’épaisseur variable, est renárquablement bien réalisée par les Pediastrum (f. 30) appartenant aux Chlorophycées; leurs colonies discoïdes, appclées coenobes, peuvent être pleines au perforées (clathrées) : $P$. duplex -(f. 37). Les cellules marginales de ces coenobes sont pourvues d'appendices latéraux plus ou moins saillants. L'on rencontre écralement, dans ce même groupe des Chlorophycées, des formes en coenobes discoïdes chez les Crucigenia (f. 38), Tetrastrum (f. 39). Enfin, parmi les Chrysomonadinées, Cyclonexis annularis (f. 40), a un coenobe en forme d'auge arrondie.

$2^{\circ}$ La forme tabulaire est représentée chez les Algues bleues par les Merismopedia (f. 41), à cellules réunies également sur un seul plan, formant ainsi des colonies subcarrées au contour récrulier, ou comme Holopedia irregularis (f. 42), autre Cyanophy̨te, à surface irrégulièe.

Il existe aussi une forme parallèle aux Merismopedic chez plusieurs Bactéries planctoniques: Lampropedia hyalina (f. 13) et Thiopedia rosea (f. 44). L'on retrouve également la forme tabulaire régulière dans les Volvocales avec le Gonium pectorale (f. 45).

$3^{\circ}$ La forme en filet, quo l'on rencontre chez des Algues très différentes: Algues bleues Cyanodictyon (f. 46), Algues vertes Hydrodictyon ou filet d'eau (f. 47). Lon connaît unc forme parallèle non planctonique chez les Bactériacées : Thiodictyon et Pedodiclyon.

$4^{\circ}$ La forme en cordon ou en bande à deux dimensions caractéristiques des Fragilaria (f. 48) chez les Diatomóes, présente dans son grand axe une torsion en spirale; ce type d'association se retrouve ćọalement chez les Desmidiaćes avec les genres Iyalotheca, Spondylosum, Onychonema, 'Sphaerozosma, Gymnozyga, Desmidium (f. 49), dont les longs cordons subissent également une torsion. Enfin chez les Protococcales, certains Scenedesmus présentent, à un moindre derrré, cette même forme. Les Chrysomonadinées offrent une association semblable avec Chlorodesmus hispidus (f. 50 ).

Les cellules qui composent les associations coloniales précédentes sont en forme de baguette chez les Fragilaria, en cellules aplaties pour les Desmidiacées, en fuseau ou en table chez plusieurs Scenedesmus (f. 51).

$5^{\circ}$ La forme en étoile; celle-ci résulte d'une tendance chez certaines Algues en baguettes réunies à leur base par un coussinet grélatineux, de se ranger sur un même plan. Ce dispositif se trowe réalisé chez lęs Diatomées planctoniques par les Asterionella (f. 52). Cette même forme se rencontre dans les Algues vertes avec Actinastrum Hantzschii (f. 53) ; fréquemment dans ce dernier exemple, les éléments de la colonie ne sont pas tous appliqués sur un même plan, majs dirigés dans divers sens et formant ainsi une sphère étoilée.

$6^{\circ}$ La forme en chaîne dans laquelle les unités cellulaires du type baguette sont rangées bout à bout et en grand nombre, en se suivant les unes les autres en zig-zagr, est réalisé en particulier par les Dialoma (f. 54).

(A suivre). 


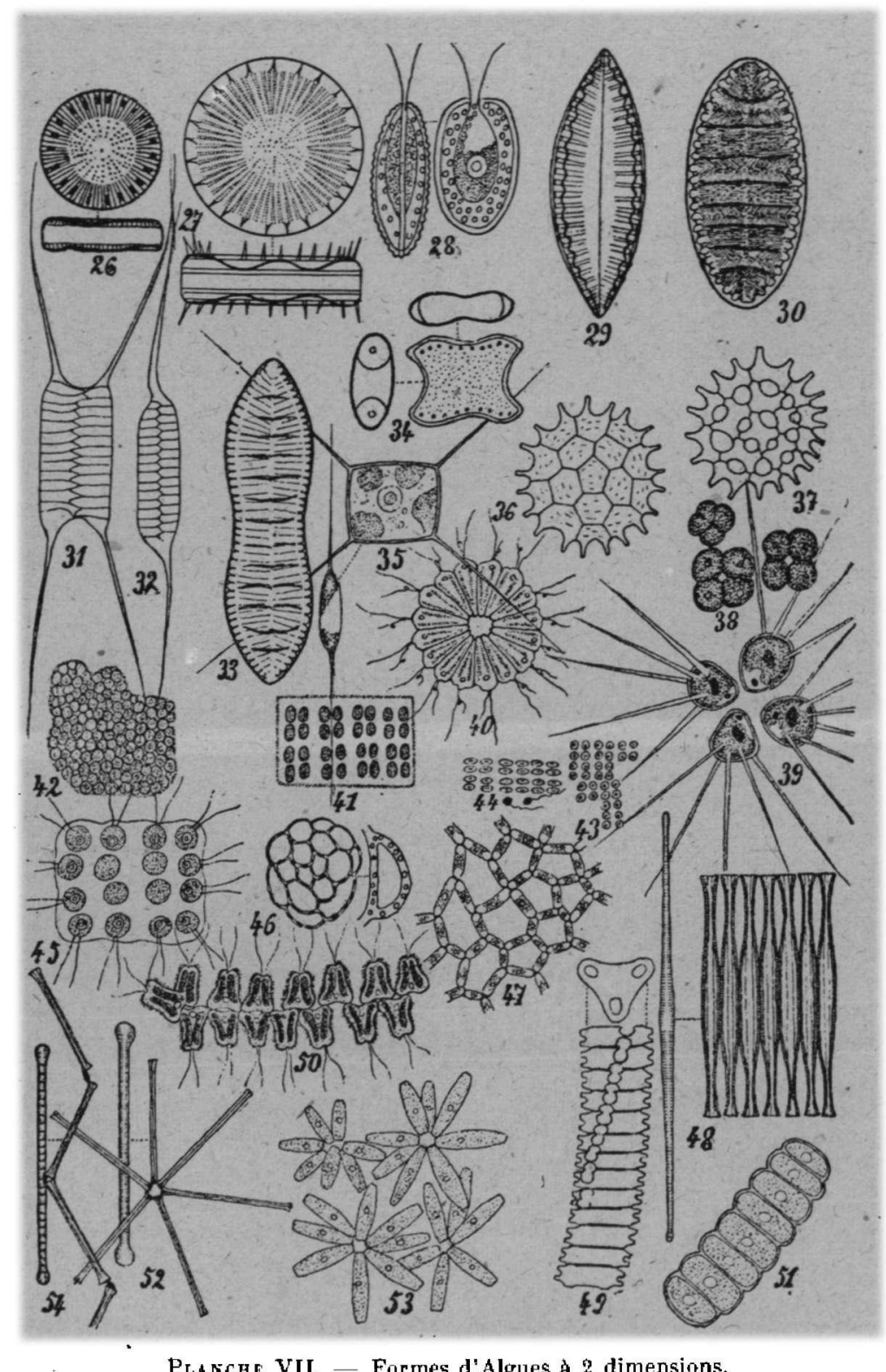

Planche VII. - Formes d'Algues à 2 dimensions.

A. - Type disque: - figs. 26 Cyclotella comta (Ehr.) Kütz.; -- 27 Stephanodiscus astrea (Ehr.) Grun.

B. - Type levticulathe et autros:- figs. 28 Phacotus lenticularis Stein; - 29 Surirella biseriata Bréb., var. bifrons (Ehr.) Hust.; - 30 Cymatopleura elliptica (Bréb.) W. Sm.; - 31 Attheya Zachariasi Brun ; - 32 Rhizosolenia eriensis H. L. Sm.; - 33 Cymatopleura solea (Bréb.) W. Sm.; - 34 Tetraëdron platyisthmum (Arch.) G. S. West; - 35. Pseudotetraëdron neglectum Pascher.

C. - Typf disque pluai-cellulaire : - figs. 36 Pediastrum Boryanum (Turp.) Menegh.; - 57 P. duplex Meyen; - 38 Crucigenia quadrata Morren;-39 Tetrastrum multisetum (Schmidle) Chod.; - 40.Cyclonexis annularis Stokes.

D. - Type tabUlaire: - figs. 41 Merismopedia glauca (Ehr.) Naeg.; - 12 Holopedia geminata Lagerh.; - 43 Lampropedia hyalina J. Schroter; - 44 Thiopedia rosea Winogr.; - 45 Gonium pectorale Miill.

E. - TYPE FLLT : - figs. 46 Cyanodictyon reticulatum (Lemm.) Geitler; - 27 Hydrodictyon reticulatum (L.) Lagerh.

F, - TyP CORDON oU BANDE:- figs. 48 Fragilaria crotonensis Kitton;- 49 Desmidium Swartzü Ag.; - 50 Chlorodesmus hispidus Phillips; - 51 Scenedesmus bijuga (Turpin) Lagerh.

G. - TYpe Étotle : - figs. 52 Asterionella gracillima (Hantz.) Heiberg; - 53 Actinastrum Hantzschü Lagerh.

H. - TYPE CHAINe : - fig. 54 Diatoma elongatum (Lyngb.) Ag. 